\section{NSAID-Unverträglichkeit: Meloxicam als Alternative}

\author{
Mit der generellen Empfehlung an Patienten mit einer allergisch \\ oder pseudo-allergisch bedingten Analgetika-Unverträglichkeit, \\ diese Stoffe doch zu meiden, ist vielen Betroffenen nur bedingt \\ geholfen. Als Ausweichpräparate könnten sich die neuen, \\ spezifisch das Isoenzym 2 der Cyclooxygenase hemmende \\ Analgetika wie Meloxicam anbieten.
}

Im Vordergrund der Nebenwirkungen nicht-steroidaler Analgeti$\mathrm{ka} /$ Antiphlogistika (NSAIDs) steht die Magen-Darm-Unverträglichkeit. Nicht selten sind aber auch Hauterscheinungen, die allergisch, pseudo-allergisch oder toxisch bedingt sein können. Verglichen mit echten Analgetika-Allergien, deren Häufigkeit auf etwa 5\% aller Reaktionen unter NSAIDs geschätzt wird, sind Pseudo-Allergien ohne einen immunologischen Nachweis z.B. in Form von spezifischem IgE die häufigste Ursache. Solche Reaktionen werden vor allem unter Acetylsalicylsäure (ASS) gesehen und treten gehäuft bei Patienten mit chronischer Urtikaria oder einer chronischen Rhinosinusitis (Polyposis) mit oder ohne Asthma auf.

\section{Analgetika-Enthaltsamkeit nicht immer praktikabel}

Die allergologische Diagnose solcher Analgetika-Intoleranzen in der täglichen Praxis ist in der Regel frustran vor allem, wenn die Patienten eine Pseudo-Allergie haben. Und häufig läßt sich der allgemeine Rat an die Patienten, doch in Zukunft auf NSAIDs generell $\mathrm{zu}$ verzichten und ggf. auf stärker wirksame, zentral angreifende Analgetika wie Tramadol zurückzugreifen, nicht realisieren.

Mit der Entwicklung neuer Analgetika, die relativ spezifisch die Typ-2Isoform der Cyclooxygenase (COX) hemmen, bestand bei Allergologen die Hoffnung, mit diesen NSAIDs möglicherweise eine gute Ausweichmedika-

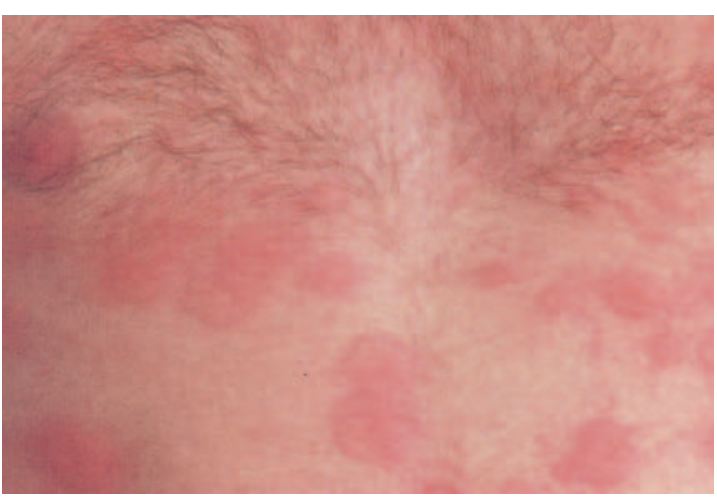

Urtikaria am Stamm mit münzgroßen, geröteten Quaddeln. Hautreaktionen sind häufig ein Symptom von Arzneimittelallergien, z.B. gegen nicht-steroidale Antiphlogistika/Antirheumatika (NSAIDs).

tion für Patienten mit Analgetika-Intoleranz in der Hand zu haben. Dies scheint sich zu bestätigen.

Wie D. Quarantino, Rom/Italien auf dem diesjährigen Treffen der European Academy of Allergology an Clinical Immunology berichtete, wurde der COX-2-Hemmer Meloxicam, der bei uns schon seit längerem unter dem Handelsnamen Mobec $^{\circledR}$ vertrieben wird, von 170 Patienten mit Unverträglichkeitsreaktionen gegen verschiedene Analgetika meist problemlos vertragen.

Von diesen Patienten hatten etwas über die Hälfte $(n=92$ ) eine singuläre Intoleranz gegenüber einem einzelnen NSAID - überwiegend gegen ASS und Pyrazolonderivaten, seltener gegen Propionsäurederivate, Diclofenac oder Paracetamol. 78 Patienten klagten über Unverträglichkeiten gegen mehrere NSAIDs, wobei vor allem die Kombinationen ASS/Pyrazolone, ASS/Pyr-
azolone/Paracetamol und ASS/Paracetamol genannt wurden.

Die Symptome der Patienten manifestierten sich bei über $80 \%$ an Haut oder Schleimhaut: Urtikaria und Angioödeme alleine oder in Kombination sowie Rhinitis mit und ohne Asthma. Ein Stevens-Johnson's-Syndrom oder anaphylaktische Reaktionen waren mit jeweils weniger als $2 \%$ eher selten.

Alle Patienten erhielten unter ärztlicher Aufsicht randomisiert eine kumulative Dosis von 7,5 mg Meloxicam innerhalb von einer Stunde - das entspricht der Dosierung einer Tablette Mobec ${ }^{\circledR}$ 7,5. Die Patienten wurden 6

Stunden nachbeobachtet und auf kutane oder respiratorische Reaktionen hin überprüft. Lediglich 1 Patient $(0,6 \%)$ entwickelte in dieser Zeit eine diffuse Urtikaria, alle anderen Patienten bleiben ohne Nebenwirkungen.

\section{Gute Verträglichkeit auch unter Nimesulid}

Über ähnlich positive Ergebnisse berichtete in Brüssel eine spanische Arbeitsgruppe (B. Cueva et al.) in einer Posterpräsentation. In der dabei vorgestellten, ebenfalls plazebokontrollierten Studie vertrugen 2 von 18 Patienten mit einer allergischen oder pseudo-allergischen Überempfindlichkeit gegen verschiedene NSAIDs den bei uns noch nicht zugelassenen COX-2-Inhibitor Nimesulid - in dieser Untersuchung in Mehrfachapplikation über 2 Tage gegeben.

Fazit: Die neue Gruppe der COX-2Inhibitoren, bei uns durch Meloxicam bereits im Markt vertreten, scheint bei vielen Patienten mit einer Unverträglichkeit gegenüber NSAIDs (Allergie, Pseudo-Allergie) eine verträgliche Alternative zu sein. Doch auf der ganz sicheren Seite befindet man sich mit diesem Präparat auch nicht: Bereits in seiner Oktoberausgabe 1998 berichtet das arznei-telegramm über Angioödeme und andere Hautreaktionen unter Meloxicam. Eine kritiklose Empfehlung ohne vorherige allergologische Testung ist deshalb abzulehnen. $\quad b k$

Vortrag und Poster präsentiert anläßlich des jährlichen Treffens der European Academy of Allergology an Clinical Immunology, Brüssel/Belgien, 1999. 